\title{
Detection of Masses in Digital Mammogram Using Second Order Statistics and Artificial Neural Network
}

\author{
Al Mutaz M. Abdalla', Safaai Dress ${ }^{2}$, Nazar Zaki $^{3}$ \\ ${ }^{1,2}$ Faculty of Computer Science and Info. Systems, Universiti Teknologi Malaysia, \\ Malaysia \\ Mutazhh30@yahoo.com \\ safaai@utm.my \\ ${ }^{3}$ Faculty of Information Technology, UAE University, UAE \\ nzaki@uaeu.ac
}

\begin{abstract}
When researchers are studying the detection of breast tumors, there has been great attention to the modern textural features analysis of breast tissues on mammograms. Detecting of masses in digital mammogram based on second order statistics has not been investigated in depth. During this study, the breast cancer detection was based on second order statistics. The extraction of the textural features of the segmented region of interest (ROI) is done by using gray level co-occurrence matrices (GLCM) which is extracted from four spatial orientations; horizontal, left diagonal, vertical and right diagonal corresponding to $\left(0^{\circ}, 45^{\circ}, 90^{\circ}\right.$ and $\left.135^{\circ}\right)$ and two pixel distance for three different block size windows $\left(8 \times 8,16 \times 16\right.$ and 32x32). The results show that the $G L C M$ at $0^{\circ}, 45^{\circ}, 90^{\circ}$ and $135^{\circ}$ with a window size of $8 X 8$ produces informative features to classify between masses and non-masses. Our method was able to achieve an accuracy of $91.67 \%$ sensitivity and $84.17 \%$ specificity which is comparable to what has been reported using the state-of-the-art Computer-Aided Detection system.
\end{abstract}

Keywords: Artificial Neural Network, Mammogram, Masses, GLCM

\section{Introduction}

Breast cancer is the second causes of death for women around the world. Any average woman has one chance in eight (or about 12\%) of developing breast cancer during her life. An effective way to decrease the mortality rates due to breast cancer has been accomplished by the early detection of breast cancer using the screening mammography [1] [2]. But it is still a difficult task to recognize the doubtful abnormalities in digital mammograms in spite of the increase in technology. There are several reasons for that, first; mammography provides relatively low contrast images, especially in the case of dense or heavy breasts and second; symptoms of abnormal tissue may remain quite subtle. For example, speculated masses that may indicate a malignant tissue within the breast are often difficult to detect, especially at the early stage of development [3].

The recent use of the second order statistics and machine learning (ML) classifiers has established a new research direction to detect masses in digital mammogram. The second order statistics of breast tissue on mammograms remains an issue of major importance in mass characterization. Compared to other mammographic diagnostic approaches, the mammographic has not been studied in depth because of the inherent difficulty and fuzziness [4]. In this paper, Artificial Neural Network based classifier (ANN) is proposed for tumor classification. The second order statistics is used as texture features extraction technique to be used in conjunction with ANN. This work is organized as follows. In Section 2 we present related works for detection of masses in mammogram images. In Section 3 material used is presented. In Section 4, 5 and 6 the methods for feature extraction and ANN classification are presented. In Section

DOI : $10.5121 /$ ijcsit.2011.3312 
7, the data analysis is done and the results are shown. The application of the proposed results is also shown in Section7. The last Section 8 has the conclusion and recommendations.

\section{Related Works}

A lot of research has been done on the textural analysis on mammographic images with the focus on detecting the boundary of tumors and micro-calcifications [5]. Youssry et al. [6] have proposed a method to detect masses in mammograms on the basis of texture features using five co-occurrence matrices extracted from four different angles $(0 \mathrm{o}, 45 \mathrm{o}, 90 \mathrm{o}$, and $135 \mathrm{o})$ and four pixel distance $(\mathrm{d}=1,3,6$ and9). Costaridou et al. [7] explains the k-means algorithm for image segmentation and co-occurrence matrix that is used for analysis of segmented structures, and the classification of masses using support vector machines (SVMs). Karahaliou et al. [8] proposed two methods based in detection of structures in image using GLCM obtained from a number of equal size areas surrounding the local ROI. [9] Presented a neuro-fuzzy model for fast detection of candidate circumscribed masses in mammograms and texture features are estimated using cooccurrence matrices which are used to train the neuro- fuzzy model.

[10] Proposes a Computer-Aided Detection system (CAD) constructing five co-occurrence matrices at different distances for each region of interest (ROI). A different number of statistical features are used to train and test the Radial Basis neural, the overall sensitivity of the method was found to be 0.91 .

[11] Study the differentiation of dense regions containing speculated masses from regions of normal dense tissues, by means of feature analysis on wavelet-processed mammograms. The analysis of spatial GLCM using different orientations and distances resulted in a multiresolution texture feature of second order statistics. Karahaliou et. al. [12] has applied thirteen textural features from four GLCMs to investigate whether texture properties of the tissue surrounding micro-calcifications using a wavelet spatially adaptive enhancement method (wavelet transform).

The classification step is very important for the performance of the computer aided diagnosis (CAD). The classification techniques are used to classify the region of interest in the mammogram. There are many classifiers techniques which are performed well in mass classification such as linear discriminants analysis (LDA) and artificial neural network (ANN). The medical image system uses other classifiers like binary decision tree, support vector machine (SVM) and Bayesian network.

LDA's H.D Cheng and H.P Cheng [13, 14] constructed decision boundaries by optimizing certain criteria to classify cases into one of mutually exclusive classes. They show high performance for linear separable problems but poor performance for non linear separable data.

ANNs are precious tools in many medical diagnostic systems. [15] Reviewed the improvements in health care increased from the contribution of NN in medical field. ANN is better in difficult situations for decision making with the massive amount of data as local operations, distributed representations and non-linear processing of data is very cumbersome. Thus when expert knowledge is unavailable in full-fledged sense ANN provides alternative and better solutions.

Oliveira et al. [16] have proposed a method to detect the masses by using the K-means and SVM for image segmentation and co-occurrence matrix to describe the texture of segmented structures and achieved $85 \%$ accuracy.

Therefore it is clear that the performance of CAD systems commonly depends on feature selection and training database than the classifiers. 


\section{Materials and Methods}

Digital Database for Screening Mammography (DDSM) [17] has been used to evaluate the proposed system. The database contains approximately 2,620 cases. In each of the case, two images of each breast, associated patient information like age, stage of the tumor, subtlety rating for abnormalities, American College of Radiology (ACR) breast density rating are studied. The Mammograms are digitized by various scanners depending on the source of the data.

A set of statistical texture feature functions was applied to a set of 120 digitized mammograms in specified regions of interest. In this paper we have focused on Grey Level co-occurrence matrices in four different directions this due to the fact that the Gray Level Co occurrence Matrix (GLCM) may provide the texture- context information. In the first step the digitized sample consists of 120 mammographic images originating from the Digital Database for Screening Mammography (DDSM) has been randomly selected with different age groups ranged from (35-80) to form this dataset. The selected cases contain 60 normal, 30 benign and 30 malignant digitized cases at 50 micron meter and 12 bit gray level. In the second stage the region of interest has been selected which contains the suspicious region of interest (ROI) as shown in Figure 1. In the third stage, the feature selected from the ROI and statistical texture features are calculated for each ROI. Figure 2 summarizes the main steps in texture analysis and classification. In the proceeding sections we discuss the mentioned steps in details.

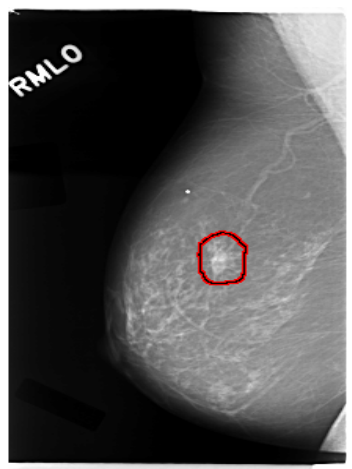

Figure 1: Digitized mammogram showing one manually segmented malignant mass.

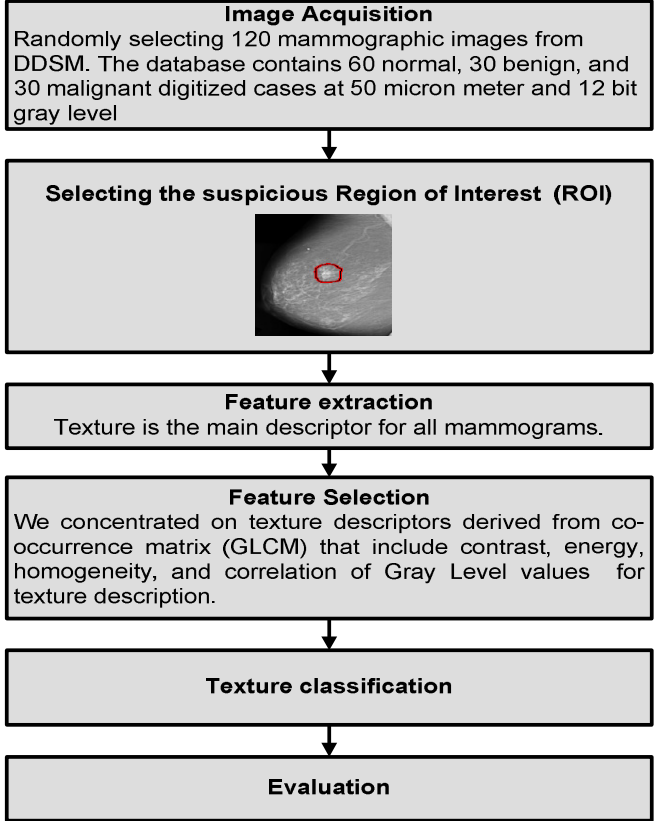

Figure 2: Diagram showing the main steps in texture analysis and classification 


\section{Feature extraction and selection}

Statistical texture features have been proven to be powerful in classifying masses and normal breast tissues [6], and the authors previous work [18]. The implementation of feature extraction procedure relies on the quality of the texture, which is the main descriptor for all the mammograms. In this work, we concentrate on statistical descriptors that include contrast, energy, homogeneity, and correlation of gray level values. Table 1 describes each of this features which are extracted from (GLCM).

Table 1: Description of texture feature derived from GLCM

\begin{tabular}{lll}
\hline $\begin{array}{l}\text { Texture descriptor } \\
\text { derive from GLCM }\end{array}$ & Formula & Description \\
\hline Contrast & $\sum_{i, j=0}^{N-1} P_{i j}(i-j)^{2}$ & $\begin{array}{l}\text { Measures of intensity contrast } \\
\text { between a pixel and its neighbor }\end{array}$ \\
Energy & $\sum_{i=1}^{N} \sum_{j=1}^{N} p(i, j)^{2}$ & $\begin{array}{l}\text { Is the sum of square elements in } \\
\text { GLCM }\end{array}$ \\
Homogeneity & $\sum_{i, j=0}^{N-1} \frac{P_{i j}}{1+(i-j)^{2}}$ & $\begin{array}{l}\text { Represent the closeness of the } \\
\text { distribution of elements in GLCM } \\
\text { to the GLCM diagonal }\end{array}$ \\
Correlation & {$\left[\sum \sum(i j) p(i, j)\right]-\mu_{x} \mu_{y}$} & $\begin{array}{l}\text { To see how correlated a pixel to } \\
\text { its neighbor over the whole image }\end{array}$ \\
\hline
\end{tabular}

\section{1. Grey Level Co-Occurrence Matrices}

The first order statistics of grey-level histograms give parameters for each processed region in the mammogram but do not provide any information about the repeating nature of texture. According to Beichel et al. [19], co-occurrence matrices describe the level of gray level configuration and are used to explain the matrices of relative frequencies. Therefore the GLCM is an advanced method of using different combinations of pixel brightness values which are the grey levels in the image.

GLCM are constructed by observing pairs of image cells distance d from each other and incrementing the matrix position corresponding to the grey level of both cells. This allows us to derive four matrices for each given distance and four different directions.

A co-occurrence matrix contains the frequency of a certain pair of pixels repetition in an image [20]. Figure 3 shows an example of how we can construct a co-occurrence matrix with eight grey levels computed using one for distance between pixels and zero degrees for the direction. In this case, the element $(1,1)$ of $\mathrm{C}$ matrix is equivalent to 1 because it has been found only once in the original image $\mathrm{f}$. Another example is shown in the same Figure of the element $(6,2)$; where there are three occurrences because a pixel with a value of 6 has a pixel valued 2 immediately to its right. The other elements of $\mathrm{C}$ are computed in the same way. 


\begin{tabular}{|c|c|c|c|c|c|c|c|c|c|c|c|c|c|c|}
\hline 1 & & 7 & 5 & 3 & 2 & & \multirow[b]{3}{*}{1} & \multirow[b]{3}{*}{2} & \multirow[b]{3}{*}{3} & \multirow{3}{*}{4} & \multirow{3}{*}{5} & \multirow{3}{*}{6} & \multirow[b]{3}{*}{7} & \multirow{3}{*}{8} \\
\hline 5 & 1 & 6 & 4 & 2 & 5 & & & & & & & & & \\
\hline 2 & 0 & 6 & 4 & 1 & 2 & & & & & & & & & \\
\hline 4 & 3 & 4 & 5 & 5 & 1 & 1 & $\frac{1}{1}$ & 2 & 0 & 0 & 0 & 1 & 1 & 0 \\
\hline 0 & 7 & 1 & 7 & 6 & 2 & 2 & 0 & 0 & 0 & 0 & 1 & 1 & 0 & 0 \\
\hline 7 & 4 & 6 & 2 & 6 & 2 & 3 & 0 & 1 & 0 & 1 & 0 & 0 & 0 & 0 \\
\hline & & $\downarrow$ & & & & 4 & 0 & 0 & 1 & 0 & 1 & 0 & 0 & 0 \\
\hline & & & & & & 5 & 2 & 0 & 1 & 0 & 1 & 0 & 0 & 0 \\
\hline & & Mat & $\operatorname{ix} f$ & & & 6 & 1 & 3 & 0 & 0 & 0 & 0 & 0 & 1 \\
\hline & & & & & & 7 & 0 & 0 & 0 & 0 & 1 & 1 & 0 & 2 \\
\hline & & & & & & 8 & 1 & 0 & 0 & 0 & 0 & 2 & 2 & 1 \\
\hline
\end{tabular}

\section{Matrix $C$}

Figure 3: Example of how to generate a Co-occurrence matrix with eight grey levels

There is $\mathrm{Ng}$ dimension of square matrix of the Grey Level Co-occurrence matrix, where the dimension $\mathrm{Ng}$ is considered to be the number of gray levels in the image which assigned values between 0 and 255 grey levels. The element $(i, j)$ of the square matrix is generated by counting the number of times a pixel with value ${ }^{i}$ is neighboring to a pixel with value $\mathrm{j}$ and then dividing the entire matrix by the total number of such comparisons made. Each entry is therefore considered to be the probability that a pixel with value $i$ will be found adjacent to a pixel of value $^{j}$. Adjacency can be defined to occur in four directions in a $2 \mathrm{D}$, square pixel image as shown in Figure 4.

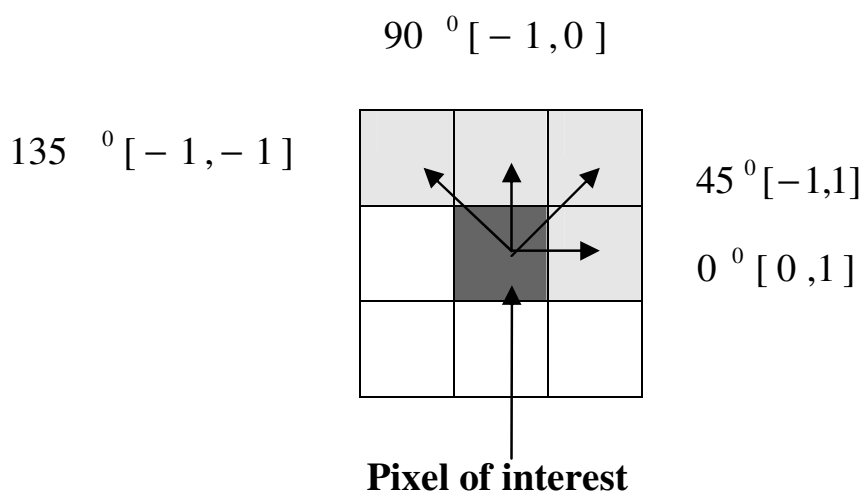

Figure 4: Adjacency of pixel in four directions (horizontal, vertical, left and right diagonals)

In this case we have used windows of several sizes $(8 \times 8,16 \times 16$ and $32 \times 32)$ because the largest masses area within a $32 \times 32$ window and the smallest is within $8 \times 8$ windows. The segmentation using a window is important because it will determine the ability of the texture descriptor to differentiate between the masses and the non-masses areas. 


\section{Artificial Neural Network}

An Artificial Neural Network (ANN) is an information processing model that is stimulated by the way biological nervous systems, such as the brain, process information. The main component of this model is the structure and the development of the information processing system.

It is composed of a large number of highly consistent processing elements called (neurons) working in unity to solve specific problems. ANNs, like people, learn by example. An ANN is configured for a specific application, such as pattern recognition or data classification, through a learning process. Learning in biological systems involves adjustments to the synaptic connections that exist between the neurones [22].

\subsection{Multi-layer Perceptron (MLP)}

Multi-layer perceptron (MLP) is a very common neural network model. It is a highly structured hierarchy of perceptrons which are used for training and training methods.MLP structure consists of three layers - input, hidden and output layers [21]. The development and the training of such a network is more complicated and is a result of teamwork of many single perceptrons. When an error occurs in the output, its is very difficult to state the error came from which node or which layer of the network and how much adjustment is to be done with the weights. The solution to such problems is done by finding the effect of all the weights in the network. This is done by the back-propagation algorithm which is based on the least mean square algorithm.

The nodes of the MLP system whether input, hidden or the output nodes are well connected with variable weights in the forward feed connections. The output is calculated based on the target output. The total mean square error (MSE) shown in equation 1, is based on the training patterns of the calculated and target outputs [21].

$$
\mathrm{MSE}=\frac{1}{2} \sum_{j=1}^{m} \sum_{i=1}^{k}\left(T_{i j}-O_{i j}\right)^{2} \ldots
$$

Where $\mathrm{m}$ is the number of examples in the training set, $\mathrm{k}$ is the number of output units, ${ }^{i j}$ is the target output value (either 0.1 or 0.9 ) of the $i^{\text {th }}$ output unit for the $j^{\text {th }}$ training example, and $O_{i j}$ is the actual real-valued output of the $i^{\text {th }}$ output unit for the $j^{\text {th }}$ training example.

The MSE between the calculated output and the target output is minimized by the iterative gradient technique that uses the back-propagation algorithm. The training process is initialized by setting some small random weights. The neural network is presented with some training data and the weights are adjusted till the MSE can be reduced to an acceptable level. A neural network has the ability to extract patterns and detect trends that are too complex to be noticed by either humans or other computer techniques [21].

\section{Proposed Methods}

The Second Order Statistics and ANN have been exploits in major mammographic characteristics to classify the mass into benign or malignant. To realize the objectives of this paper ANN uses the texture descriptor derived from GLCM contrast, energy, homogeneity, and correlation of grey level values. These 4 features are used in preparing the training data for multi-layer perceptron (MLP) neural network which are obtained from the whole extracted region of interest. The calculated four features and their corresponding target values (for benign $=0$ and malignant $=1$ ) are stored in a file. The same process is repeated for more masses both malignant and benign. This way all the training samples are stored in the file that is used as inputs to the ANN to train the network to produce the weights needed for testing the classifier. 
The training samples are used to train the network and the number of input units, hidden units and output unit are entered into the system. The proposed artificial neural network formation is shown in Table 2. Since four features are used in the application the number of input units (n) is therefore equal to 4 . The output in this case is either benign or malignant which can be represented by 1 unit. Hidden units are calculated using the formula shown in equation 2 . The weights vector can be calculated by using the formula presented in equation 3 .

$$
\begin{aligned}
& \text { Hidden Units }=(n+1) * \frac{2}{3} \ldots \ldots \ldots \ldots . .2 \\
& \text { Weights }=n *\left((n+1) * \frac{2}{3}\right)+(n+1) * \frac{2}{3} \ldots 3
\end{aligned}
$$

Once the training is done, the weight vector is used to test the proposed ANN. In that case weights are kept constant. Since weights are not changing during testing it is called offline processing.

Table 2: Proposed ANN structure for mass classification

\begin{tabular}{ll}
\hline \hline Input Units & $\mathrm{N}=4$ (Texture descriptor derived from GLCM \\
Hidden Units & 3 (Calculated from equation 2$)$ \\
Output Unit & Either 0 or 1 (can be represented by 1 unit) \\
Weights & 10 (calculated from equation 3$)$ \\
\hline \hline
\end{tabular}

\section{Results and Discussion}

In this paper we used a set of 120 digitized mammograms, $25 \%$ (30 out of 120) set of image are used for training, and 75\% (90 out of 120) are used for testing purpose. Table 3 shows the proposed ANN composition which has three layers comprised of four units in input layer, three hidden units and one output unit. The total weights in this case are 10. Three values of window or block sizes are applied in this experiment, which are $8 \times 8,16 \times 16$ and $32 \times 32$ pixels. The overall classification for benign is $84.53 \%$ for the window $8 \times 8$, and classification for malignant is $91.81 \%$. Window size $8 \times 8$ shows good classification in comparison to $16 \times 16$ and $32 \times 32$ pixels. In Table 3 we show the accuracy for all the three window sizes 8x8 16x16 and 32x32.

Table 3: Mass classification by window size

\begin{tabular}{ccccc}
\hline \hline & \multicolumn{2}{c}{ Correct Classification } & \multicolumn{2}{c}{ Misclassification } \\
\hline \hline Window 8x8 & Benign & Malignant & Benign & Malignant \\
\hline Window 16x16 & $84.17 \%$ & $91.67 \%$ & $15.83 \%$ & $8.33 \%$ \\
Window 32x32 & $81.67 \%$ & $89.17 \%$ & $18.33 \%$ & $10.83 \%$ \\
\hline \hline
\end{tabular}

The performance of the system is evaluated in terms of specificity and sensitivity. 
The performance of the binary classification is either positive or negative and is the statistical measures based on specificity and sensitivity. Sensitivity is a probability that is a measure of actual positive proportion or tumourous growth. The equation of sensitivity is explained in equation 4. Specificity is defined in the specificity measures and the measure of negatives which are used to benign masses explained in equation 5 .

$$
\begin{gathered}
\frac{T P}{T P+F N} \ldots \ldots \ldots . .4 \\
\frac{T N}{T N+F P} \ldots \ldots .5
\end{gathered}
$$

These two measures are closely related to Type I and II errors. $100 \%$ sensitivity is the theoretical optimal prediction for the tumourous growth. 100\% specificity is the theoretical optimal prediction for the non tumourous growth. The sensitivity and specificity of the proposed system is shown in Table 4.

Where $\mathrm{TP}=$ True positive, $\mathrm{TN}=$ True negative, $\mathrm{FP}=$ False positive, and $\mathrm{FN}=$ False negative.

Table 4 showing the comparison of sensitivity and specificity of the proposed system

\begin{tabular}{|l|c|c|c|c|c|c|}
\hline Windows & TP & TN & FP & FN & Sensitivity (\%) & Specificity (\%) \\
\hline Window 8x8 & 110 & 101 & 19 & 10 & 91.67 & 84.17 \\
\hline Window 16x16 & 107 & 98 & 22 & 13 & 89.17 & 81.67 \\
\hline \hline Window 32x32 & 106 & 96 & 24 & 14 & 88.33 & 80.00 \\
\hline
\end{tabular}

In Figures 5 -7 detailed window sizes comparisons are presented.

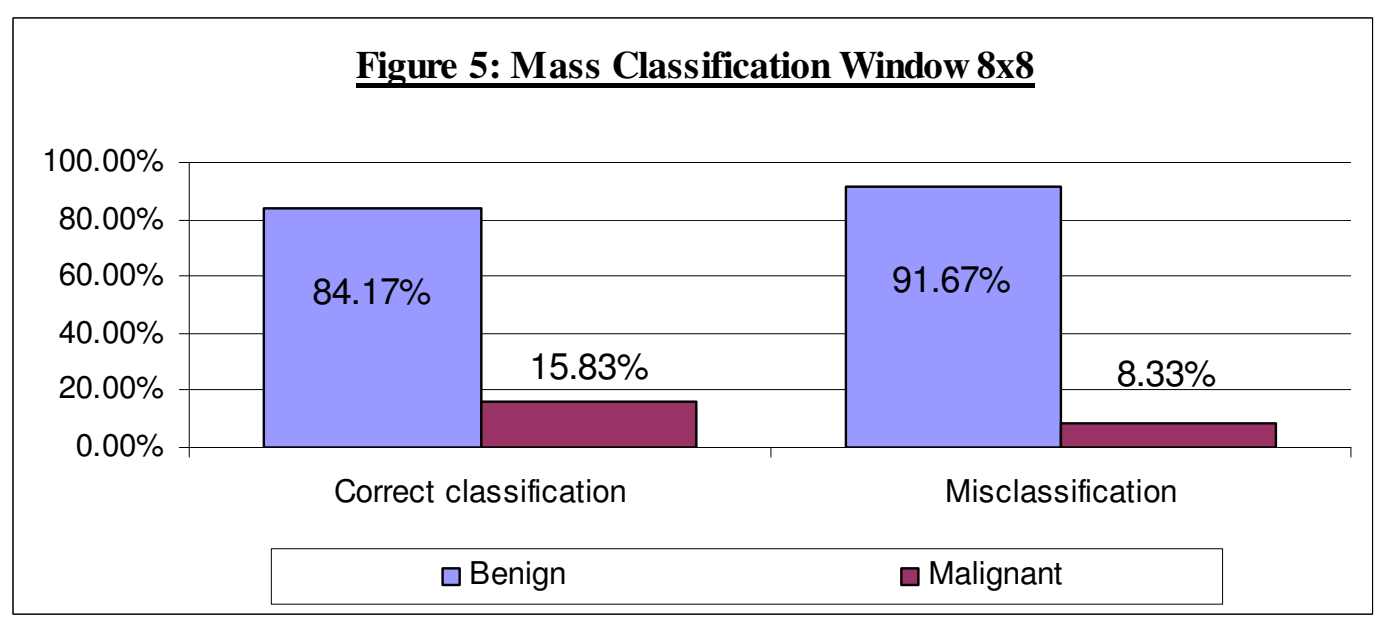



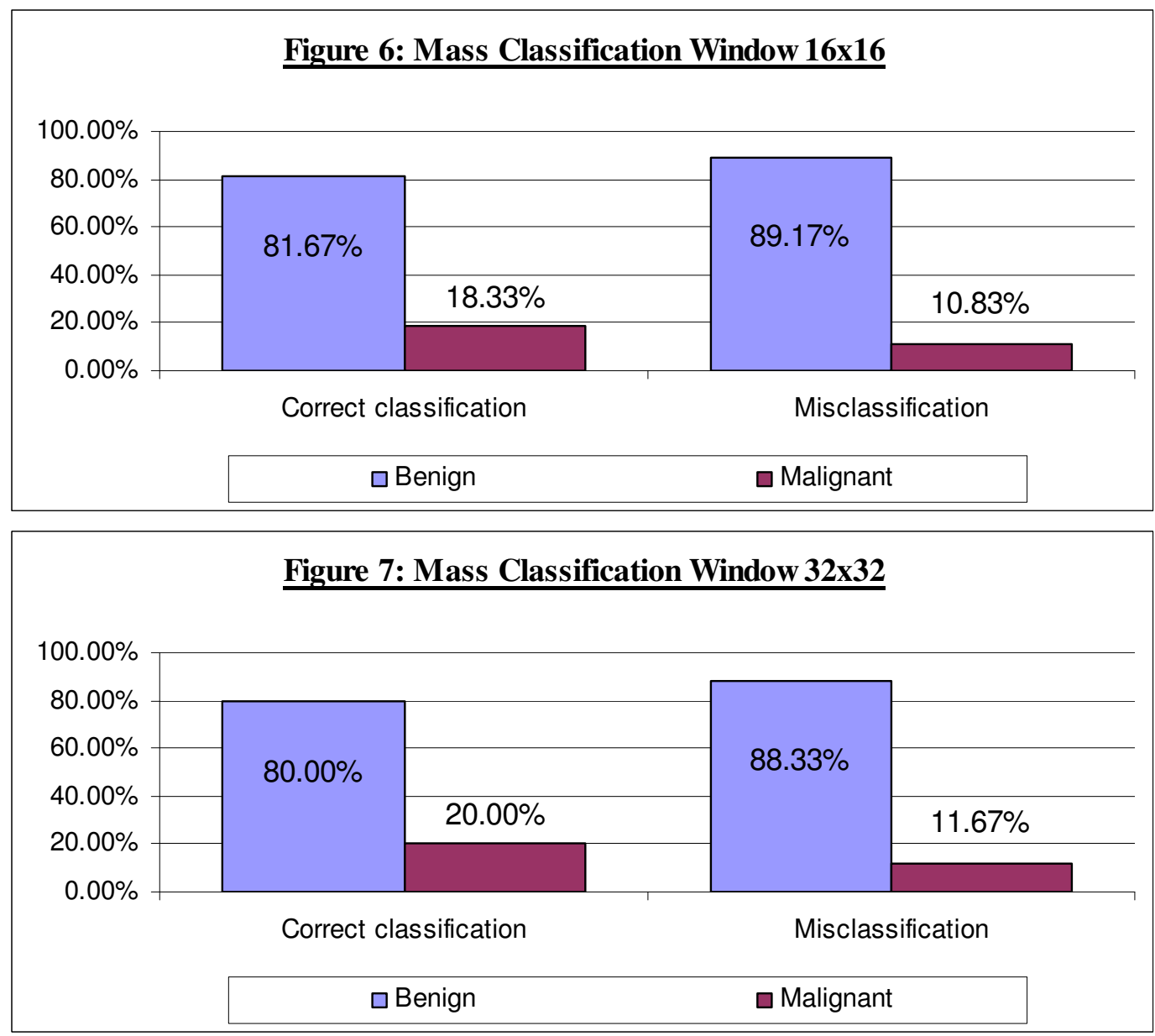

Graphs 8 and 9 shows the accuracy and comparison for the three window sizes, in window size $8 \times 8$ the sensitivity is $91.67 \%$, in $16 \times 16$ is $89.17 \%$, and in $32 \times 32$ is $88.33 \%$. While the specificity for window size $8 \times 8$ is $81.17 \%$, in $16 \times 16$ is 81.67 , and in $16 \times 16$ is $80.00 \%$. It has been found that our method was able to achieve good accuracy with the window size $8 \times 8$.

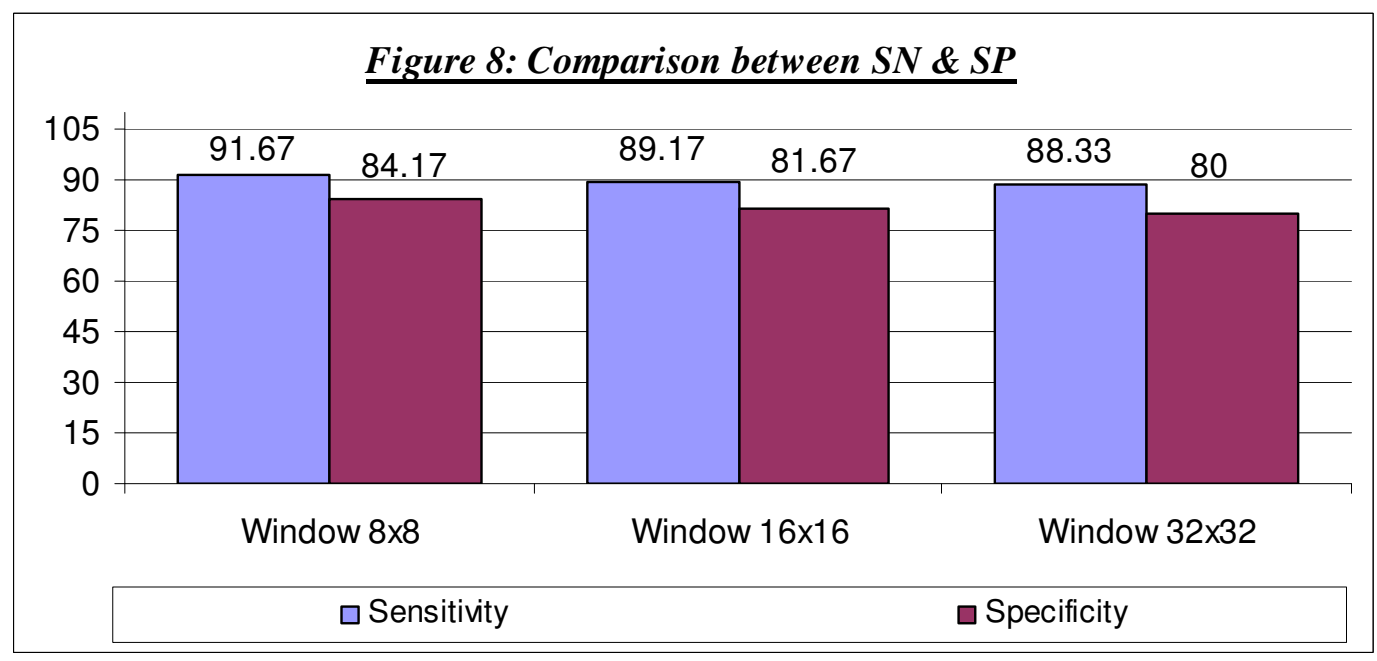




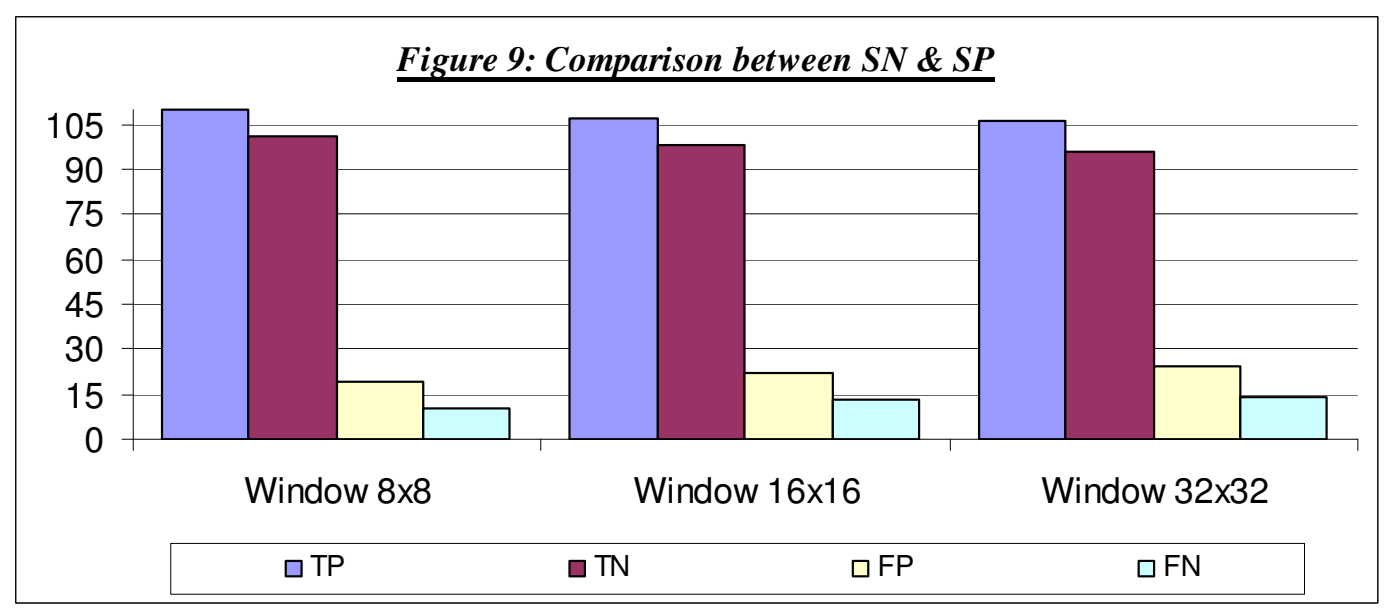

\section{Conclusions}

ANN and second order statistics shows very significance performance in classifying the masses into benign and malignant. The performance of the proposed system is evaluated by using the sensitivity and specificity using 120 images containing normal, benign, and malignant cases with different size and shape. The result achieved with block size window 8x8 compared to other windows and shows that $91.67 \%$ sensitivity, and $84.17 \%$ specificity. This work has been successfully found that the properties of GLCM with conjunction with the ANN can be applied to detect masses and non-masses in digital mammogram.

\section{References}

[1] Smith,R.A., "Screening women aged 40-49: where are we today?" J Natl Cancer Inst, 1995, pp. 1198-1199.

[2] Tabar,L., Fagerber,G., and Chen,R.H., "Efficacy of breast screening by age: new results from the swedish two country trial", Cancer, 1995, pp. 1412-1419.

[3] Arodź,T., Kurdziel,M., Sevre,E.O.D., and Yuen,D.A., "Pattern Recognition Techniques for Automatic Detection of Suspicious-looking Anomalies in Mammograms", Computer Methods and Programs in Biomedicine, Elsevier, 2005, pp. 135-149.

[4] Mavroforakis,M.E.,Georgiou,H.V.,Dimitropoulos,N., Cavouras,D., and Theodoridis,S., "Mammographic masses characterization based on localized texture and dataset fractal analysis using linear, neural and support vector machine classifiers", Artificial Intelligence in Medicine, 2006, pp. 145-162

[5] Lisboa,P.G., "A review of evidence of health benefit form artificial neural networks", Neural Networks, 2002, pp. 11-39.

[6] K. Bovis and S. Singh. Detection of masses in mammograms using texture features. 15th International Conference on Pattern Recognition (ICPR'00), 2:2267, 2000.

[7] Leonardo de Oliveira Martins, Geraldo Braz Junior, Arist'ofanes Correa Silva, Anselmo Cardoso de Paiva, and Marcelo Gattass. Detection of Masses in Digital Mammograms using Kmeans and Support Vector Machine: Electronic Letters on Computer Vision and Image Analysis 8(2):39-50, 2009

[8] L. Blot and R. Zwiggelaar. Extracting background texture in mammographic images: Cooccurrence matrices based approach. Proceedings of the 5th International Workshop on Digital Mammography, Toronto (Canada), pages 142\{148, 2000. 
[9] N. Youssry, F.E.Z. Abou-Chadi, and A.M. El-Sayad. Early detection of masses in digitized mammograms using texture features and neuro-fuzzy model. 4th Annual IEEE Conf on Information Technology Applications in Biomedicine, 2003.

[10] M. Jirari. A computer aided detection system for digital mammograms based on radial basis functions and feature extraction techniques. IEEE Engineering in Medicine and Biology 27th Annual Conference, 2005.

[11]L. Costaridou, P.N. Sakellaropoulos, M.A. Kristalli, S.G. Skiadopoulos, A.N. Karahaliou, I.S. Boniatis, and G.S. Panayiotakis. Multiresolution feature analysis for differentiation of breast masses from normal tissue. 1st International Conference on Experiments/Process/System Modelling/Simulation/Optimization, 2005.

[12] A. Karahaliou, I. Boniatis, P. Sakellaropoulos, S. Skiadopoulos, G. Panayiotakis, and L. Costaridou. Can texture of tissue surrounding microcalci ${ }^{-}$cations in mammography be used for breast cancer diagnosis? Nuclear Instruments and Methods in Physics Research a 580, pages $1071\{1074,2007$.

[13]H.D. Cheng, X.J. Shi, R. Min, L.M. Hu, X.P. Cai, H.N. Du (2006) “Approaches for automated detection and classification of masses in mammograms", Pattern Recognition, Vol. 39, pp. 646668.

[14]H.P. Chan, D. Wei, M.A. Helvie, B. Sahiner et.al. (1995) "Computer-aided classification of mammographic masses and normal tissue: linear discriminant analysis in texture feature space", Journal of Physics in Medicine and Biology, Vol. 40, pp. 857-876.

[15] P.J.G. Lisboa, (2000) "A review of evidence of health benefits from artificial neural networks in medical intervention”, Neural Networks, Vol. 15, pp. 11-39.

[16]L. D. Oliveira, Geraldo B. Junior, A.C. Silva, and Marcelo Gattass. Detection of Masses in Digital Mammograms using K-means and Support Vector Machine. Electronic Letters on Computer Vision and Image Analysis 8(2):39-50, 2009

[17] http://marathon.csee.usf.edu/Mammography/Database.html

[18] Al Mutaz M. Abdalla, Safaai Deris, Nazar Zaki and Doaa M. Ghoneim (2007), "Breast Cancer Detection Based on Statistical Textural Features Classification", 4th International Conference on Innovations in Information Technology, Dubai 18-20 Nov 2007 IEEE.

[19] R. Beichel and M. Sonka. Computer vision approaches to medical image analysis. Lecture Notes in Computer Science, Springer, 4241, 2006.

[20] Haralick,R.M., Shanmugam,K., Dinstein,I., “Textural features for image classification”, IEEE Trans Sys Man Cyb, 1973, pp. 610-21.

[21] M. J. Islam, Majid Ahmadi, Maher A. Sid Ahmed. (2010) "An Efficient Automatic Mass Classification Method In Digitized Mammogram Using Artificial Neural Network” International Journal of Artificial Intelligence \& Application, vol.1, No.3,

[22]Christos Stergiou and Dimitrios Siganos. Neural Network, Available on http://www.doc.ic.ac.uk/ nd/surprise_96/journal/vol4/cs11/report.html 